\title{
Striated muscle-derived intraspinal choristoma: A case report
}

\author{
PENG-FEI CHANG ${ }^{1}$, LI-MEI QU ${ }^{2},{\text { MING } \text { LI }^{1} \text { and BIN QI }}^{1}$ \\ Departments of ${ }^{1}$ Neurosurgery and ${ }^{2}$ Pathology, The First Hospital of Jilin University, Changchun, Jilin 130021, P.R. China
}

Received April 29, 2016; Accepted March 17, 2017

DOI: $10.3892 / 01.2017 .6302$

\begin{abstract}
Choristoma is a mass of tissue that is histologically normal for the organ or part of the body from which it originates, but is located at an abnormal site. Choristoma of the nervous system involves the occurrence of residual dysplastic tissues outside the nervous system and exhibits a low incidence. Thus far, there has been no prior report of intraspinal choristoma originating from the striated muscle. The patient in the present case was a young man who was admitted to the hospital wing to intermittent lumbago. A lumbar magnetic resonance imaging examination revealed a cystic-solid lesion in the spinal canal at the S1 level. Preoperatively, the lesion was suspected to be a teratoma and was therefore completely removed. The postoperative pathological report suggested that the mass was an intraspinal choristoma, a benign lesion associated with a good prognosis, which had originated from striated muscle. Early diagnosis and treatment can prevent progression of nerve dysfunction. The patient recovered well postoperatively and the symptoms of lower back pain disappeared. Although intraspinal choristomas are rare, when a lesion in the spinal canal is diagnosed, it should be considered in the differential diagnosis tumors.
\end{abstract}

\section{Introduction}

Choristoma of the nervous system is characterized by the occurrence of residual dysplastic tissues outside of the nervous system (1-4). Intraspinal choristomas are rare and, to the best of our knowledge, only three cases have been reported (2-4). The most likely differential diagnoses for spinal choristoma include lipoma, hamartoma and teratoma. Spinal hamartomas contain mostly mature and well differentiated tissue from ectodermal and mesodermal layers. Alternatively, if tissues from all 3 germ layers are identified in the lesion, a teratoma with malignant potential should be considered. The mechanism for the formation of spinal choristoma is not well understood.

Correspondence to: Dr Bin Qi, Department of Neurosurgery, The First Hospital of Jilin University, 72 Xinmin Street, Changchun, Jilin 130021, P.R. China

E-mail: qibinqi@126.com

Key words: intraspinal, choristoma, pathology, diagnosis
The present case involved a striated muscle-derived choristoma, which was located in the lumbosacral spinal canal. This presentation is rare and has not been previously reported, to the best of our knowledge. The present report discusses the clinical manifestations, imaging features, pathological changes and differential diagnosis of the patient and reviews the associated literature. Based on the findings, a striated muscle-derived choristoma was diagnosed. Written informed consent was obtained from the patient for the publication of this study.

\section{Case report}

A 26-year-old man was admitted to the Department of Neurosurgery at The First Hospital of Jilin University, (Changchun, China) on 3rd July 2011 with intermittent lumbago that had persisted for 3 days. A physical examination indicated percussive pain in the lower back but no symptoms in neurological examinations. A lumbar magnetic resonance imaging (MRI) scan and a Gadolinium-enhanced scan revealed a low-lying, enlarged terminal filament, with the terminal end located at the L5 level and a broadened vertebral canal at the S1 level. An ovoid cystic-solid lesion, $\sim 2.8 \times 2.0 \mathrm{~cm}$ in size, was visible at the end of the terminal filament. High T1-weighted image (T1WI) and T2WI signals were present in the solid portion of the lesion, with decreased signals in a fat-saturated scan, and slightly lower T1WI signals and high T2WI and fat-saturated signals in the cystic portion of the lesion (Fig. 1). The enhancement scan showed that the vertebral canal was broader at the S1 level. Mixed cystic and solid lesions were observed at the filum terminale; the solid portion exhibited hyper-intensity, whereas the cystic portion exhibited slight hypo-intensity. The clinical diagnosis was of a sacral spinal lesion at the S1 level (possibly a large teratoma) and tethered cord syndrome. Surgery was conducted under neurophysiological monitoring. The resected intraspinal tumor was gray with moderate harness, lacked an abundant blood supply and evidently adhered to the caudaequina. The tumor was removed from the caudaequina and completely resected with KUSA ultrasonic aspirator (Sonastar FS-1000-RF; Misonix, Inc., Farmingdale, NY, USA); the total size was $\sim 3.0 \times 2.5 \times 2.0 \mathrm{~cm}$. The pathological examination (hematoxylin and eosin staining on formalin-fixed, $3 \mu \mathrm{m}$-thick sections) indicated the presence of mature striated muscle, fat, scattered nerve and glandular-like tissue without cell atypia (Fig. 2).

The expression of carcinoembryonic antigen (CEA), creatine kinase $(\mathrm{CK})$, epithelial membrane antigen (EMA), 

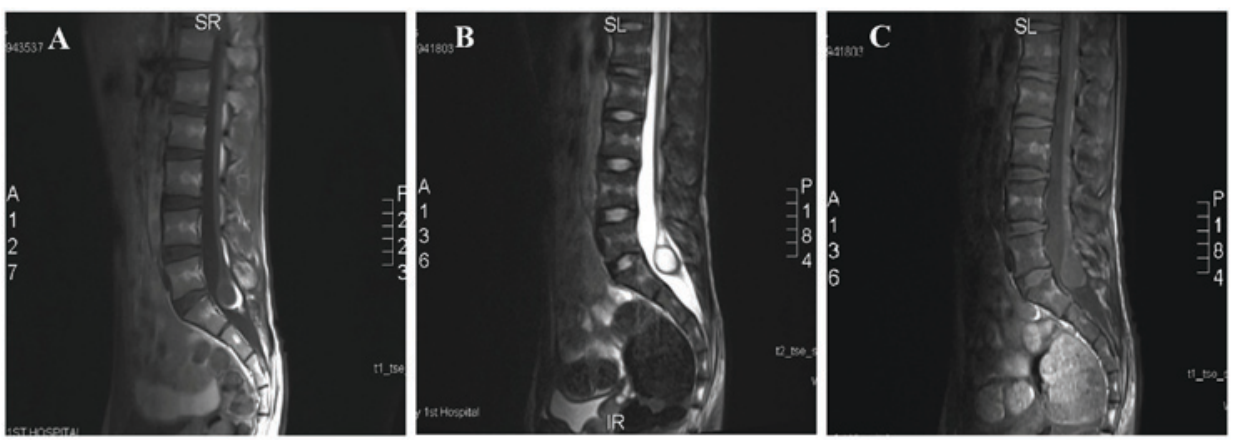

Figure 1. MRI and contrast-enhanced MRI of the lower torso of the patient. (A) Sagittal T1-weighted lumbar MRI scan, (B) Sagittal T2-weighted lumbar MRI scan and (C) a contrast-enhanced scan showing: A low-lying, enlarged terminal filament with the terminal end located at the L5 level; a broadened vertebra canal at the S1 level; an ovoid cystic-solid lesion $\sim 2.8 \times 2.0 \mathrm{~cm}$ that is visible at the end of the terminal filament; high T1WI and T2WI signals in the solid portion of the lesion; decreased signals in a fat-saturated scan; and slightly lower T1W1 signal and increased T2WI and fat-saturated signals in the cystic portion of the lesion. The enhanced scan shows that the vertebral canal is broader at the S1 level. Mixed cystic and solid lesions are observed at the filum terminale, and the solid portion shows hyper-intensity, while the cystic portion shows slight hypo-intensity. MRI, magnetic resonance imaging; T1WI, T1-weighted image.

glial fibrillary acidic protein (GFAP), S-100, Vimentin and neurofilament (NF) protein were detected by immunohistochemistry using the EnVision method (1). Antibodies included CEA (cat. no. 2A-0063), CK (cat. no. 2M-0067), EMA (cat. no. ZM-0095), GFAP (cat. no. 2A-0529), S-100 (cat. no. 2M-0224), Vimentin (cat. no. 2M-0260) (all from ZSGB-BIO, Beijing, China) and NF (cat. no. MAB-0134) (Maixin Biological Co., Fuzhou, China). All antibodies were diluted at a ratio of 1:100.

The immunohistochemical staining results were as follows: CEA (glandular- +), CK (glandular +), EMA (glandular- +), GFAP (-), S-100 (focal +), Vimentin (glandular- -), and NF (focal +) (Fig. 3). The final diagnosis was intraspinal choristoma at the $\mathrm{S} 1$ level. The patient recovered well postoperatively and the lower back pain disappeared; however, the patient succumbed to injuries sustained in a traffic accident 6 months after discharge.

\section{Discussion}

Choristoma of the nervous system is characterized by the occurrence of residual dysplastic tissues outside the nervous system in multiple systems and organs, including the head, eyes, tongue, limbs, esophagus, stomach and hepatic arterial trunk (2). Choristomas grow slowly and are usually asymptomatic, with dysfunctions only occurring once the size of the tumor increases. The majority of choristomas of the central nervous system are derived from glial cells, including astrocytes and oligodendrocytes, neurons, axons, the ependyma and the choroid plexus, and are without evident mitosis or tumor-like blood vessels. The first case involving multiple nests of neuroglial tissue in the meninges of the spinal cord was reported by Wolbach (3). Kurman et al (4) identified the first case of choristoma derived from the lumbosacral region. Chen et al (5) reported the existence of a growing mammary choristomain a girl at puberty, which resembled a lumbosacral lipomyelomeningocele. Neuromuscular choristoma of the oculomotor nerve has also been reported $(1,6)$. The present case involved a striated muscle-derived choristoma located in the lumbosacral spinal canal, which contained striated muscle, and adipose, nerve and glandular tissues.

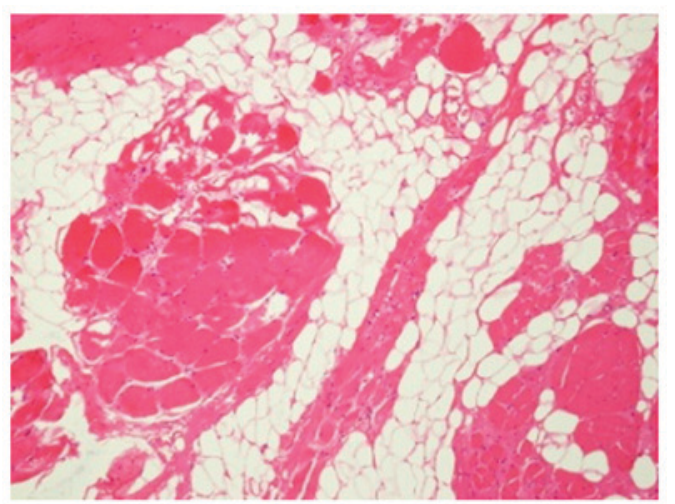

Figure 2. Pathological hematoxylin and eosin staining of a section of the choristoma showing mature striated muscle tissue, fat tissue, scattered nerve tissue and glandular-like tissue without cell atypia. Magnification, x200.
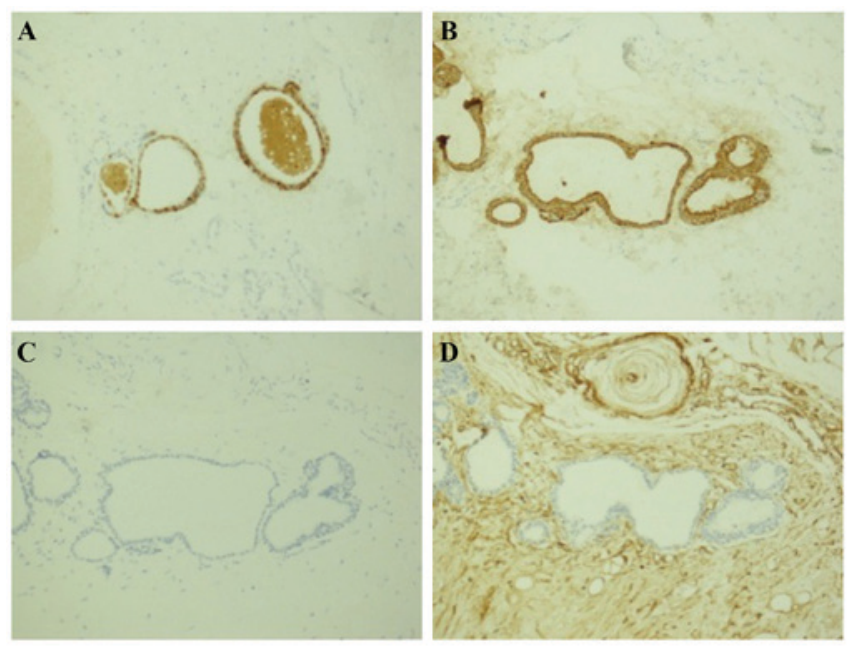

Figure 3. Immunohistochemical staining of the choristoma, showing immunohistochemical staining for (A) carcinoembryonic antigen in glandular-like tissue, (B) cytokeratin in the glandular tissue, (C) glial fibrillary acidic protein (no staining) and (D) vimentin (focal staining). Magnification, x200.

This type of lesion is extremely rare and has not been previously reported. 
The pathogenesis of choristoma is currently unclear for the following reasons. First, the protrusion hypothesis proposed by Cooper and Kernohan (7) states that lesions protrude from pre-existing defects of the mature brain or spinal cord. Second, the migration hypothesis by Gyure et al (8) states that abnormal embryonic neural epithelium migrates to the subarachnoid space and differentiates from weeks 5 to 6 of the embryonic stage. Third, the separation hypothesis by Harris et al (9) states that two outer valgi of the primitive brain are isolated from brain precursors and exhibit dysplasia during the early stages of neurogenesis or brain vesicle formation. However, the pathogenesis of choristoma and the origin of the ectopic tissue in the lumbosacral spinal region have not yet been elucidated.

The differential diagnosis was ofteratomaor hamartoma. Midline spinal cord teratomas are tumors derived from the three germinal layers and generally contain cartilage, squamous cells, skin appendages, cholesterol and other components, including columnar mucosa and spinal nerves. MRI examinations of teratomas reveal hyper-intense fat, isointense soft tissue and hypo-intense calcification, generally without enhancement (of these findings, calcification is particularly important) $(10,11)$. In the present case, the patient's tumor consisted of striated muscle and gland tissue; therefore, a diagnosis of teratoma could be excluded. A spinal cord hamartoma is composed of well-differentiated tissues from the endoderm and ectoderm, in which loose connective tissue and mucoid mesenchyme differentiate into mature connective tissue, cartilage and fat (12-14). A hamartoma is a benign lesion, similar to a choristoma. The two can be differentiated as follows: A hamartoma is an excess of normal tissue in a normal situation, while a choristoma is an excess of tissue in an abnormal situation (5).

The clinical neurological symptoms of intraspinal choristoma vary according to the location and size of the lesions (2-4). The patient exhibited no symptoms in neurological examinations other than intermittent back pain. For patients with a tethered spinal cord, surgical intervention should be performed to prevent progressive dysfunction of the spinal cord, as this condition interferes with normal metabolism and can lead to progressive ischemia and nerve damage $(3,4)$. An intraspinal choristoma is a benign lesion with a good prognosis, for which the early diagnosis and treatment can prevent the progression of nerve dysfunction $(3,4)$. The patient recovered well following surgery and the lower back pain symptoms disappeared. Intraspinal choristomas are exceedingly rare lesions. Preoperative diagnosis of intraspinal choristoma is difficult and surgical specimens are usually required to confirm the diagnosis.

\section{References}

1. Boyaci S, Moray M, Aksoy K and Sav A: Intraocular neuromuscular choristoma: A case report and literature review. Neurosurgery 68: E551-E555, 2011.

2. Krolls SO, Jacoway JR and Alexander WN: Osseous choristomas (osteomas) of intraoral soft tissue. Oral Surg Oral Med Oral Pathol 32: 588-595, 1971.

3. Wolbach SB: Congenital rhabdomyoma of the heart. J Med Res 16: 495-520.7, 1907.

4. Kurman RJ, Funk RL and Kirshenbaum AH: Spinal bifida with associated choristoma of Müllerian origin. J Pathol 99: 324-327, 1969.

5. Chen X, Harter J, Iskandar BJ and Salamat MS: Growing mammary choristoma masquerading as a lumbosacral lipomyelomeningocele in a pubertal girl. J Neurosurg Pediatr 8: 321-324, 2011.

6. Kawamoto S, Matsuda H, Ueki K, Okada Y and Kim P: Neuromuscular choristoma of the oculomotor nerve: Case report. Neurosurgery 60: E777-E778, 2007.

7. Cooper IS and Kernohan JW: Heterotopic glial nests in the subarachnoid space; histopathologic characteristics, mode of origin and relation to meningeal gliomas. J Neuropathol Exp Neurol 10: 16-29, 1951.

8. Gyure KA, Morrison AL and Jones RV: Intracranial extracerebral neuroglial heterotopia: A case report and review of the literature. Ann Diagn Pathol 3: 182-186, 1999.

9. Harris CP, Townsend JJ and Klatt EC: Accessory brains (extracerebral heterotopias): Unusual prenatal intracranial mass lesions. J Child Neurol 9: 386-389, 1994.

10. Castillo M, Smith MM and Armao D: Midline spinal cord hamartomas: MR imaging features of two patients. AJNR Am J Neuroradiol 20: 1169-1171, 1999.

11. Brownlee RD, Clark AW, Sevick RJ and Myles ST: Symptomatic hamartoma of the spinal cord associated with neurofibromatosis type 1. Case report. J Neurosurg 88: 1099-1103, 1998.

12. Hader WJ, Steinbok P, Poskitt K and Hendson G: Intramedullary spinal teratoma and diastematomyelia. Case report and review of the literature. Pediatr Neurosurg 30: 140-145, 1999.

13. Ghostine S, Perry E, Vaynman S, Raghavan R, Tong KA, Samudrala S, Johnson JP and Colohan A: The rare case of an intramedullary cervical spinal cord teratoma in an elderly adult: Case report and literature review. Spine (Phila Pa 1976) 34: E973-E978, 2009.

14. Borlot F, Soares MS, Espíndola AA, Reed UC, Matushita H and Teixeira MJ: Intramedullary spinal teratoma: A rare condition with a good outcome. Arq Neuropsiquiatr 67 (3A): 733-735, 2009. 